\title{
REDUCIENDO LA CARGA DE ENFERMEDAD GENERADA POR EL CONSUMO DE ALCOHOL EN EL PERÚ: PROPUESTAS BASADAS EN EVIDENCIA
}

\author{
Fabián Fiestas $1,2, a$
}

\begin{abstract}
RESUMEN
El consumo de alcohol constituye uno de los más importantes factores de riesgo para mala salud y muerte prematura en el Perú. Son necesarias medidas que disminuyan o controlen el gran impacto que tiene el alcohol en la sociedad peruana. El presente artículo identifica y promueve el uso de medidas de salud pública que cuentan con evidencia científica sólida de efectividad y, en algunos casos, costo-efectividad. Al ser apoyadas por la evidencia científica, las diez medidas aquí identificadas representan un conjunto de medidas con alta probabilidad de éxito. Se recomienda que los gobiernos, nacionales o locales, tomen estas medidas no aisladamente sino en combinación, en un plan u hoja de ruta que establezca en forma contextualizada el engranaje en que se aplicarán. Tomando en cuenta los recursos que se tienen, algunas de estas medidas podrían implementarse a corto o mediano plazo, mientras que las otras lo serían a más largo plazo.
\end{abstract}

Palabras clave: Consumo de bebidas alcohólicas; Enfermedad; Factores de riesgo; Perú (fuente: DeCS BIREME).

\section{REDUCING THE BURDEN OF DISEASE CAUSED BY ALCOHOL USE IN PERU: EVIDENCE- BASED APPROACHES}

\begin{abstract}
Alcohol use is one the most important risk factors for illness and early death in Peru. Measures aimed at decreasing or controlling the great impact caused by alcohol in the Peruvian society are urgently needed. This article identifies and promotes the implementation of public health measures supported by sound scientific evidence of effectiveness or, in some cases, cost-effectiveness. The 10 evidence-based public health measures identified and described here represent a set if measures with high probability of success if implemented, as they are supported by scientific evidence. We recommend that governments, at the national or local levels, apply these measures not individually, but in combination, arranging them into a plan or roadmap, where the framework in which they will be applied must be established according to each context. Considering the available resources, some of these measures could be implemented in the short and medium term while the others can be set in the long-term.
\end{abstract}

Key words: Alcohol drinking; Disease; Risk factors; Peru (source: MeSH NLM).

\section{EL PROBLEMA DEL ALCOHOL}

El consumo de alcohol es una prioridad de salud pública global y regional, pues está entre los factores de riesgo que más pérdidas económicas ocasionan a las sociedades por razones de salud, además del sufrimiento que causa a individuos y familias. En el mundo, el uso de alcohol explica más muertes que el SIDA o la tuberculosis, con un $4 \%$ del total de años perdidos por muerte prematura, siendo este impacto más intenso para los varones entre 15 y 45 años ${ }^{(1)}$.

En el Perú, el abuso y la dependencia al alcohol figuran como la tercera causa más importante de pérdidas económicas por muerte prematura o discapacidad, superados solo por la neumonía y los accidentes de tránsito ${ }^{(2)}$. Sin embargo, la contribución del consumo de alcohol puede ser aun mayor si tomamos en cuenta que este es también causa de una variedad de otros problemas de salud, como accidentes de tránsito (los cuales son la primera causa de discapacidad y muerte prematura en nuestro país), violencia, accidentes caseros y de trabajo, uso de drogas ilegales e involucramiento en conducta sexual de riesgo ${ }^{(3)}$. Además, el consumo crónico de alcohol ha sido consistentemente asociado con un mayor riesgo de otras enfermedades como depresión mayor (4), enfermedades cardiovasculares (5) y el cáncer ${ }^{(6)}$. Asimismo, se ha encontrado que las personas

${ }^{1}$ Unidad de Análisis y Generación de Evidencias en Salud Pública, Instituto Nacional de Salud. Lima, Perú

2 Red para la Acción y Avance de la Salud Mental y Psiquiatría (Red AVANSE-PSI). Lima, Perú

${ }^{a}$ Médico epidemiólogo

* Este artículo se basa en la nota técnica "medidas de salud para el control de uso de alcohol", publicada en la página web de la Unidad de Análisis y Generación de Evidencias en Salud Pública, Instituto Nacional de Salud.

Recibido: 08-01-12 Aprobado: 22-02-12 
con consumo crónico de alcohol tienen peor pronóstico en enfermedades como la neumonía ${ }^{(7)}$, el VIH/SIDA ${ }^{(8)}$ y la tuberculosis ${ }^{(9)}$, tanto por el efecto inmunosupresor del alcohol (10), como por el impacto de dicho uso en la adherencia al tratamiento para estas enfermedades ${ }^{(11)}$.

En términos económicos, según la Comisión Nacional para el Desarrollo y Vida sin Drogas (DEVIDA), el problema del alcohol le genera al Perú pérdidas anuales de al menos 245 millones de dólares ${ }^{(12)}$. La Organización Panamericana de la Salud ha informado que en nuestro país se consume aproximadamente 9,9 litros de alcohol puro per capita por año, en la población adulta (de 15 años o más), lo cual coloca al Perú entre los países latinoamericanos con mayor consumo ${ }^{(3)}$. De las bebidas registradas, la que más se consume es la cerveza $(88 \%)$, seguida por otros licores y, con menos frecuencia, los vinos ${ }^{(3)}$.

Se ha estimado que actualmente hay aproximadamente 1,5 millones de personas con dependencia al alcohol en nuestro país. Sin embargo, el problema es más grande en la población general puesto que muchas personas lo consumen de manera episódica hasta la intoxicación (borrachera) lo cual las pone en riesgo de accidentes de todo tipo, incluidos los automovilísticos, a infecciones de transmisión sexual y a sufrir lesiones como consecuencia de actos de violencia. Una de las características más penosas del consumo agudo de alcohol es que sus consecuencias no solo atañen a aquellos que lo consumen, sino que también frecuentemente alcanza a otras personas, como sucede en los casos de choques vehiculares, atropellos, homicidios, violencia doméstica, entre otros eventos adversos que el alcohol ocasiona para la vida y la salud física y mental de las personas ${ }^{(3)}$.

Debido a las graves consecuencias ocasionadas por el alcohol a la economía, la salud y el bienestar de las personas y la sociedad en general, los gobiernos e instituciones de diversas regiones del mundo han buscado implementar políticas que ayuden a controlarlas; y es precisamente ello lo que debe hacer el Perú. Aunque se han tomado algunas medidas, estas son, en su mayor parte, aisladas, circunscritas geográficamente, insuficientes y muchas son de efectividad desconocida.

Pero la selección de políticas o intervenciones para enfrentar el problema del alcohol tiene que ser entre aquellas que tienen evidencia científica de ser costoefectivas y sostenibles ${ }^{(13,14)}$. El hacerlo así, definitivamente, aumentará la probabilidad de éxito, logrando que los pocos recursos con los que se cuentan sean bien invertidos.

Así, el objetivo de este artículo es señalar desde la literatura científica mundial, aquellas medidas de salud pública que cuentan con evidencia científica sólida de efectividad o costo-efectividad, respecto a controlar los daños asociados con el uso de alcohol en las sociedades, como son los accidentes de tránsito y de otros tipos, la violencia, las lesiones físicas, enfermedades crónicas, muerte, trastornos neuropsiquiátricos, entre otros. Además, este artículo indica medidas que, aun cuando han sido ampliamente usadas en varios países, incluido el nuestro, no cuentan con sustento científico que apoye su efectividad y que, por lo tanto, no se hubiera recomendado que sean implementadas desde el punto de vista de la Salud Pública.

Esto cobra especial importancia porque en Salud Pública, cuando se aplican medidas que no sabemos si son costoefectivas, o al menos mínimamente efectivas, dos cosas pueden suceder: o la medida no funciona y es lo mismo que no haber implementado nada o la medida tiene un efecto paradójico y termina causando más daño que no haberla aplicado. En el primer caso, el perjuicio a la sociedad va en el sentido de un malgasto de los pocos recursos con los que un país como el Perú cuenta y, en el segundo caso, además del malgasto de recursos, se podría estar añadiendo más morbilidad o mortalidad a la sociedad. Esto hace extremadamente importante que cada medida de salud pública que se implemente sea respaldada por la mejor evidencia científica de efectividad o costo-efectividad posible, aun cuando esta no haya sido obtenida en el contexto local, en cuyo caso debe darse una continua producción de investigación local. Además es importante que cada medida que se aplique, aun cuando tenga evidencia que la respalde, sea supervisada y monitoreada cercanamente en el tiempo.

\section{DIEZ OPCIONES PARA ABORDAR EL PROBLEMA}

Luego de una búsqueda exhaustiva en Pubmed/ Medline, Web of Science, Google Scholar, Scielo y páginas web de instituciones de salud internacionales, como la Organización Mundial de Salud, la Organización Panamericana de la Salud, The Community Guide, entre otras, que identificó revisiones sistemáticas, metanálisis, policy-briefs e informes institucionales, se detectaron diez medidas de salud que muestran suficiente evidencia científica que reducen los daños en la sociedad producidos por el alcohol, incluyendo la violencia, accidentes de todo tipo, accidentes de tránsito, lesiones, visitas a salas de emergencia, uso no saludable de alcohol, trastornos neuropsiquiátricos, conducta sexual, abuso y dependencia al alcohol, intentos de suicidio, cirrosis y muerte.

Estas diez medidas mencionadas a continuación representan entonces oportunidades confiables para la acción, que luego podrían ser engranadas en una "hoja 
de ruta" que aquellos profesionales que ejercen cargos de toma de decisiones puedan seguir para disminuir efectiva y sostenidamente los daños que el alcohol produce en las sociedades.

\section{REDUCIR LAS HORAS DE VENTA DE BEBIDAS ALCOHÓLICAS}

La regulación de las horas de venta de bebidas alcohólicas, conocida localmente como "Plan Zanahoria" o la "Hora Segura," consiste en reducir el acceso a bebidas alcohólicas, especialmente en altas horas de la noche, a personas que ya se encuentran bajo los efectos del alcohol y tienen poca capacidad de control para detener el consumo de alcohol o para tomar medidas preventivas y no sufrir, por ejemplo, accidentes de tránsito o eventos de violencia.

La evidencia científica muestra que el restringir en, al menos, dos horas la venta de alcohol por las noches, reduce los niveles de consumo de alcohol y los daños asociados con su consumo. Además, se muestra que este efecto beneficioso es mayor cuando la restricción es en locales donde se vende y se consume bebidas alcohólicas (bares, peñas, discotecas, restaurantes, entre otros) que cuando se implementa solo en locales dedicados a la venta de dichas bebidas, como son por ejemplo los supermercados, tiendas y las licorerías ${ }^{(15-18)}$.

En estudios primarios y revisiones sistemáticas, se ha encontrado que la extensión de la restricción de dos a cuatro horas en locales de venta y consumo de alcohol, disminuye los niveles de consumo por persona y se reduce la frecuencia de lesiones por accidentes de tránsito, las admisiones en salas de emergencia de hospitales, las lesiones físicas y la violencia interpersonal, y la frecuencia de conductores ebrios. Sin embargo, si la restricción es de menos de dos horas no hay mayor efecto en la ocurrencia de eventos adversos causados por el consumo agudo de alcohol ${ }^{(16)}$. Si, por ejemplo, la restricción significa disminuir una hora el límite en que se permite la venta de bebidas alcohólicas (de las 4.00 pasar a las $3.00 \mathrm{~h}$ ), la reducción de las consecuencias del alcohol es mínima, por lo que sería una medida inefectiva. Para que la restricción tenga efecto tangible entonces, la restricción debe ser de dos o más horas (por ejemplo, de las 4.00 pasar a las $2.00 \mathrm{~h}$ ).

Bajo este conocimiento, la mayoría de ciudades en el mundo que han adoptado la restricción horaria de venta nocturna de alcohol tienen como hora límite de venta de alcohol las $2.00 \mathrm{~h}$, acompañando esta medida con un sistema estricto de vigilancia de que esta norma se cumpla, con fuertes sanciones administrativas para los infractores. Además, para evitar la movilización, especialmente en vehículos, a altas horas de la noche, de lugares con más restricción horaria a otros con menor restricción o supervisión, por personas que ya están bajo los efectos del alcohol, las normas de restricción horaria son establecidas de manera generalizada en regiones geopolíticas enteras. Por ello, si una política como esta se va a implementar en el Perú, debería ser de carácter departamental e incluso nacional.

Un caso emblemático, donde se ha podido constatar el efecto positivo que tiene el restringir el horario de estipendio de bebidas alcohólicas en reducir las consecuencias del consumo de alcohol, es el caso de la ciudad brasilera de Diadema. Esta ciudad situada a $20 \mathrm{~km}$ de la ciudad de Sao Paulo, tenía la tasa más alta de homicidios en Brasil, llegando a ser en 1999 tan alta como 103 homicidios cada 100000 habitantes, de los cuales el $65 \%$ estaban relacionados con el alcohol. En este contexto, y a la luz de estudios observacionales e informes policiales que mostraban que la mayoría de asesinatos y de asaltos a mujeres ocurrían dentro de los bares o cerca de ellos, especialmente entre las 23.00 y las $6.00 \mathrm{~h}$, el alcalde instituyó en el 2002 una ordenanza municipal que prohibía el funcionamiento de los bares después de las $23.00 \mathrm{~h}$, siendo que estos funcionaban las 24 horas del día. Con esta medida, Diadema logró reducir en $44 \%$ las muertes por homicidio que hubieran sucedido en los siguientes 3 años si no se hubiera implementado esta restricción horaria en los bares. De igual manera, la frecuencia de asaltos contra la mujer en dicho periodo disminuyó en $17 \%{ }^{(18)}$.

\section{REDUCIR LOS DÍAS DE VENTA DE BEBIDAS ALCOHÓLICAS}

Esta iniciativa consiste en establecer uno o más días de la semana en los cuales no se permite la venta de bebidas alcohólicas. En la mayoría de las ciudades donde se aplica esta norma, se ha elegido el domingo como el día en que no se puede vender alcohol, ello sucede, por ejemplo, en catorce estados de los Estados Unidos (http://www.alcoholpolicy.niaaa.nih.gov/Bans on Off-Premises Sunday Sales.html).

Existe evidencia científica sólida que sostiene que la prohibición de venta en al menos un día a la semana reduce el nivel de consumo per cápita de alcohol, así como la tasa de conductores ebrios, las atenciones hospitalarias por lesiones físicas y las muertes por accidentes de tránsito. Esta eficacia se da tanto si la medida se aplica a locales donde se consume alcohol como en locales donde solo se vende ${ }^{(19)}$.

En estudios de costo-efectividad, se encontró que en EE. UU., Canadá y Cuba por cada USD 700 invertidos 
en aplicar esta medida se prevenía un año perdido por discapacidad o muerte prematura. Debido a que una persona promedio gana al año mucho más de USD 700, entonces se evidencia que esta medida es altamente costo-efectiva (20). En el estado de Nuevo México se encontró que el dejar sin efecto la prohibición de vender bebidas empaquetadas durante los días domingo produjo aproximadamente 42 muertes adicionales en esos días en el lapso de cinco años, lo cual significó pérdidas adicionales en 6 millones de dólares por año por gastos médicos y pérdida de productividad (21).

\section{REDUCIR LA CANTIDAD DE PUNTOS DE VENTA DE BEBIDAS ALCOHÓLICAS}

Esta medida implica la regulación de la cantidad de locales de venta de alcohol (por ejemplo, el número de locales por área geográfica), la cual tiene el objetivo de reducir el número de puntos de venta de bebidas alcohólicas o limitar la apertura de nuevos puntos. Específicamente, esta medida se logra a través del manejo y control de otorgación de licencias o de zonificación para vender dichas bebidas.

La evidencia sugiere que esta medida puede causar una reducción de hasta $56 \%$ en los niveles de consumo de alcohol y en los niveles de violencia; por ejemplo, se estima que por cada seis lugares de venta que se reduzcan en un área geográfica específica se puede llegar a prevenir una víctima de violencia que necesite hospitalización. Asimismo, esta medida puede llegar a prevenir hasta $14 \%$ de accidentes automovilísticos $(22,23)$.

\section{ESTABLECER RESPONSABILIDAD LEGAL PARA EL VENDEDOR DE BEBIDAS ALCOHÓLICAS POR LOS DAÑOS OCASIONADOS POR EL CONSUMIDOR}

Esta medida consiste en hacer que los dueños de establecimientos de venta de alcohol compartan responsabilidad legal de los daños (muerte o accidentes, por ejemplo) que el consumidor ocasione como consecuencia de haber bebido alcohol en dichos establecimientos.

Esta medida ha mostrado que disminuye las muertes por accidentes de tránsito en $6,4 \%$, y la frecuencia de accidentes nocturnos de un solo vehículo en $6,6 \%$. Además, disminuye la frecuencia de casos de violencia y de enfermedades crónicas asociadas con el alcohol (24). Debido a la sólida evidencia científica que la apoya, esta medida es altamente recomendada, especialmente para disminuir accidentes de tránsito relacionados con el alcohol ${ }^{(25)}$.

\section{INCREMENTAR IMPUESTOS}

Esta medida implica el aumento del precio del alcohol, la reducción de los daños asociados al consumo de bebidas alcohólicas y el aumento de los ingresos al Estado de manera proporcional al consumo. Estas medidas consisten usualmente en imponer mayores impuestos en la cantidad de bebida comprada (y no en el precio de venta), y requieren ajustarse regularmente por el nivel de inflación anual.

Aumentar el precio del alcohol en $10 \%$ disminuye en $7,7 \%$ el nivel de consumo de bebidas alcohólicas en la población general, con un efecto mayor entre los jóvenes que son quienes tienen mayor prevalencia de consumo problemático de alcohol. De igual manera, un aumento del $10 \%$ en el precio del alcohol disminuye los accidentes de tránsito relacionados al alcohol en $12 \%$, la tasa de conductores ebrios disminuye en 5 a $8 \%$, la mortalidad debida a cirrosis en $9 \%$ y la mortalidad debida a cualquier causa en $4 \%$, aproximadamente ${ }^{(26)}$. Por el nivel de evidencia científica, esta medida es altamente recomendada (27). Sin embargo, el incremento de impuestos debe ir acompañada de medidas que vigilen y controlen estrictamente la comercialización de alcohol informal.

\section{SUPERVISAR QUE LAS LEYES EXISTENTES SE CUMPLAN}

Esta disposición implica mejorar el sistema de vigilancia para asegurar la aplicación de leyes o normas dirigidas a reducir el daño ocasionado por el consumo de bebidas alcohólicas, especialmente el cuidar aquellas normas que prohíben la venta de bebidas alcohólicas a menores de edad, con supervisión estrecha y sanciones administrativas y legales que sean ejemplares.

Por ejemplo, iniciar o aumentar la frecuencia de revisiones o constataciones de que la ley se cumpla, el aplicar severas sanciones legales o administrativas para aquellos establecimientos infractores son medidas que disminuyen los niveles de consumo de alcohol en los jóvenes. Además, estas medidas aumentan su impacto si se acompañan de estrategias que incrementan la percepción, por parte de los dueños de los locales de venta de bebidas alcohólicas, sobre el riesgo que significa para su establecimiento si no cumplen con las normas de venta establecidas. Las estrategias que incrementan la percepción de falta grave, se pueden hacer por medio de publicidad en medios masivos de comunicación.

En una revisión sistemática de la literatura que incluyó ocho estudios que evaluaban el impacto de estas medidas en el porcentaje de venta de bebidas alcohólicas 
a señuelos que eran (o lucían como) menores de edad, se encontró que estas medidas redujeron la venta a los menores de edad en $42 \%{ }^{(28)}$.

\section{SISTEMAS DE IDENTIFICACIÓN Y MANEJO TEMPRANO, BASADOS EN ESTABLECIMIENTOS DE SALUD}

Este sistema implica implementar medidas de identificación temprana de personas con problemas de alcohol, la aplicación de intervenciones breves y la derivación a tratamientos específicos cuando corresponda, operados desde servicios de consulta externa o salas de emergencia de hospitales o centros de atención primaria de la salud ${ }^{(29,30)}$.

Existe evidencia de que las intervenciones breves son eficaces en reducir el consumo perjudicial de alcohol y que su implementación en salas de emergencia hospitalarias se asocia con una reducción de futuras visitas a dichos servicios en los 12 meses siguientes.

En el Perú, un estudio encontró que $40 \%$ de los varones atendidos en la sala de emergencia de un hospital general en la ciudad de Lima tenían algún tipo de uso problemático de alcohol. Además, los resultados de este estudio sugieren que una manera, que podría ser costo-efectiva, de encontrar casos de consumo problemático de alcohol para luego ofrecer un manejo médico temprano y adecuado, es distinguir a todo varón que llega a la sala de emergencia por cualquier razón y que, además, tenga signos o refiera haber consumido alcohol antes de su arribo a la sala de emergencia ${ }^{(31)}$

Las salas de emergencia constituyen, entonces, una oportunidad de excepción para identificar de manera costo-efectiva a aquellos pacientes que se beneficiarían con las intervenciones breves para controlar el consumo problemático de alcohol o para identificar a aquellos que necesitan ser derivados a manejo especializado por presentar ya problemas de dependencia a dicha sustancia.

\section{EDAD MÍNIMA PARA COMPRAR ALCOHOL}

Establecer una edad mínima legal en que la persona pueda comprar alcohol limita los niveles de consumo de este, aunque no el acceso o la ingesta. Esto quiere decir que aunque esta medida no evita que los menores de edad encuentren maneras de comprar o conseguir bebidas alcohólicas y consumirlas, este tipo de medidas sí logran disminuir la cantidad y frecuencia con que los menores de edad toman alcohol. Específicamente, los estudios científicos sugieren que la reducción de la edad mínima para adquirir alcohol de un año (digamos de
18 a 17 años) incrementa en alrededor de un 10\% los accidentes de tránsito; mientras que estos accidentes se reducen en $16 \%$ si la edad de inicio se incrementa en un año (digamos, de 18 a 19 años). Sin embargo, la eficacia de estas medidas dependen de una vigilancia sostenida y severa por parte de las autoridades públicas ${ }^{(3,14)}$.

\section{RESTRINGIR PUBLICIDAD Y PATROCINIO}

La publicidad genera un ambiente propicio para el consumo de alcohol (sensación de aceptación y deseabilidad social), promueve su alto uso y amplia difusión. La evidencia muestra que el aumento en el gasto publicitario por parte de la industria del alcohol se asocia con un incremento en el consumo por personas de 12 años a más, y que a mayor exposición de un individuo a publicidad favorable al alcohol, mayor es la probabilidad de consumir más alcohol en el futuro ${ }^{(32)}$. Así, las medidas que disminuyen la exposición de las personas jóvenes a publicidad favorable al consumo de alcohol reducen el consumo hasta en $16 \%$, reducen la incidencia de casos nuevos de consumidores de alcohol en el futuro así como también reducen la incidencia de casos trastornos de uso de alcohol o problemas de salud asociados al alcohol, por ejemplo, accidentes, violencia o cirrosis ${ }^{(14,33,34)}$. Esto es consistente con la evidencia que muestra que la probabilidad de desarrollo de abuso o dependencia al alcohol es inversamente proporcional a la edad de inicio de consumo de alcohol. Es decir, si se logra que la edad de inicio de uso de alcohol sea mayor, entonces menor será la probabilidad de tener problemas de abuso o dependencia más tarde en la vida del individuo ${ }^{(3)}$.

\section{MEDIDAS DE CONTROL DE ACCIDENTES DE TRÁNSITO}

Muchas medidas que se han implementado $y$ estudiado, tuvieron como objetivo principal el reducir los accidentes de tránsito debido al estrecho rol causal que tiene el consumo de alcohol con este tipo de accidentes. Sin embargo, estas medidas han ofrecido ganancias secundarias al reducir también otros problemas causados por el alcohol, como son la violencia interpersonal, violencia contra la pareja, accidentes diferentes a los de tránsito o problemas de salud orgánicos. Entre las medidas con fuerte evidencia científica de ser no solo efectivas, sino también costoefectivas, están el establecer límites más bajos de concentración de alcohol en sangre, el implementar un sistema de licencias graduadas para conductores novatos, el establecer niveles de concentración en sangre aun más restringidos para conductores jóvenes, y el establecer mecanismos de verificación de sobriedad o pruebas aleatorias de aliento en conductores en 
puntos estratégicos así como también, el establecer sanciones de suspensión de licencias de conducir si se detecta niveles no permitidos de alcohol ${ }^{(3,14)}$.

\section{MEDIDAS CON EVIDENCIA INSUFICIENTE O CONTRADICTORIA DE EFECTIVIDAD}

Hay varias medidas o políticas que no cuentan con sustento científico suficiente que avale su efectividad. Estas incluyen disposiciones muy conocidas en el contexto peruano, como por ejemplo la estrategia del "Amigo elegido" o conductor asignado ${ }^{(35)}$, la educación escolar y en universidades sobre los peligros que acarrea el uso de alcohol, los anuncios en servicios públicos que alertan sobre los riesgos del consumo de alcohol y las etiquetas de advertencia que se colocan en envases, cajas, locales y ambientes donde se vende o consume alcohol ${ }^{(3,14)}$. A pesar de carecer de sustento técnico, estas medidas se han implementado en diversas oportunidades en el Perú y otras partes del mundo, pero al carecer de un sustento científico de efectividad sólido, no se recomienda aplicar estas acciones como medidas para reducir el daño producido por el uso de alcohol.

\section{CONCLUSIÓN}

El uso de alcohol constituye uno de los más importantes factores de riesgo para mala salud y muerte prematura en el Perú; la carga de enfermedad que causa es mucho mayor que la ocasionada por las drogas ilegales o por otras enfermedades visibles como la tuberculosis o el cáncer. Ello nos obliga, como actores con responsabilidades en la salud de la población, a pasar a la acción con medidas que disminuyan o controlen este tremendo impacto que tiene el alcohol en las sociedades. El presente artículo identifica las principales medidas de salud pública que cuentan con evidencia científica sólida que apoyan su efectividad y, en algunos casos, su costo-efectividad. Al ser apoyadas por la evidencia científica, las diez medidas aquí identificadas representan un conjunto de disposiciones con alta probabilidad de éxito. Se recomienda que los gobiernos, nacionales o locales las tomen no aisladamente sino en combinación, en un plan u hoja de ruta que establezca en forma contextualizada el engranaje en que se aplicarán. Tomando en cuentan los recursos disponibles, tanto para la implementación como para la evaluación continua, algunas podrán ser de implementación a corto-mediano plazo, mientras que otras lo serán a más largo plazo. Finalmente, solo así, aplicando una salud pública basada en la evidencia, es que la probabilidad de éxito de las intervenciones o políticas públicas será mayor en asegurar la vida y mejorar la calidad de vida de las personas.

\section{Conflictos de interés}

El autor declara no tener conflictos de interés.

\section{REFERENCIAS BIBLIOGRÁFICAS}

1. Musayon-Oblitas F. Potencial impacto social y económico de la muerte prematura por consumo de alcohol: Estimaciones en base a datos de mortalidad. Perú, 2008. Lima: Universidad Peruana Cayetano Heredia; 2009.

2. Velásquez A. La carga de enfermedad y lesiones en el Peru y las otras prioridades del plan esencial de aseguramiento universal. Rev Peru Med Exp Salud Publica. 2009;26(2):222-31.

3. Organización Panamericana de la Salud. Alcohol y salud pública en las Américas. Washington, D.C.: OPS; 2007.

4. Fergusson DM, Boden JM, Horwood LJ. Tests of causal links between alcohol abuse or dependence and major depression. Arch Gen Psychiatry. 2009;66(3):260-6.

5. Romelsjo A, Allebeck P, Andreasson S, Leifman A. Alcohol and mortality in 50,000 swedish conscripts-No heart protective effect up to age 55. ACER. 2010;34(suppl 3):108A-A

6. Chen L, Gallicchio L, Boyd-Lindsley K, Tao XG, Robinson $\mathrm{KA}$, Lam TK, et al. Alcohol consumption and the risk of nasopharyngeal carcinoma: a systematic review. Nutr Cancer. 2009;61(1):1-15.

7. Samokhvalov AV, Irving HM, Rehm J. Alcohol consumption as a risk factor for pneumonia: a systematic review and meta-analysis. Epidemiol Infect. 2010;138(12):1789-95.

8. Shuper PA, Neuman M, Kanteres F, Baliunas D, Joharchi N, Rehm J. Causal considerations on alcohol and HIVIAIDS--a systematic review. Alcohol Alcohol. 2010;45(2):159-66.

9. Fok A, Numata Y, Schulzer M, FitzGerald MJ. Risk factors for clustering of tuberculosis cases: a systematic review of population-based molecular epidemiology studies. Int $\mathrm{J}$ Tuberc Lung Dis. 2008;12(5):480-92.

10. Laso FJ, Vaquero JM, Almeida J, Marcos M, Orfao A. Chronic alcohol consumption is associated with changes in the distribution, immunophenotype, and the inflammatory cytokine secretion profile of circulating dendritic cells. Alcohol Clin Exp Res. 2007;31(5):846-54.

11. Hendershot CS, Stoner SA, Pantalone DW, Simoni JM. Alcohol use and antiretroviral adherence: review and metaanalysis. J Acquir Immune Defic Syndr. 2009;52(2):180-202.

12. Comisión Nacional para el Desarrollo y Vida sin Drogas (DEVIDA). Impacto social y económico del consumo de drogas en el Perú-2010. Lima: DEVIDA; 2010.

13. Fiestas F. Traducción de la investigación en acciones específicas en salud [carta]. Rev Peru Med Exp Salud Publica. 2010;27(3):478-9.

14. Anderson P, Chisholm D, Fuhr DC. Effectiveness and cost-effectiveness of policies and programmes to reduce the harm caused by alcohol. Lancet. 2009;373(9682):2234-46.

15. Task Force on Community Preventive Services. Recommendations on maintaining limits on days and hours of sale of alcoholic beverages to prevent excessive alcohol consumption and related harms. Am J Prev Med. 2010;39(6):605-6. 
16. Hahn RA, Kuzara JL, Elder R, Brewer R, Chattopadhyay S, Fielding J, et al. Effectiveness of policies restricting hours of alcohol sales in preventing excessive alcohol consumption and related harms. Am J Prev Med. 2010;39(6):590-604.

17. Douglas M. Restriction of the hours of sale of alcohol in a small community: a beneficial impact. Aust N Z J Public Health. 1998;22(6):714-9.

18. Duailibi S, Ponicki W, Grube J, Pinsky I, Laranjeira R, Raw $M$. The effect of restricting opening hours on alcohol-related violence. Am J Public Health. 2007;97(12):2276-80.

19. Middleton JC, Hahn RA, Kuzara JL, Elder R, Brewer R, Chattopadhyay S, et al. Effectiveness of policies maintaining or restricting days of alcohol sales on excessive alcohol consumption and related harms. Am J Prev Med. 2010;39(6):575-89.

20. Sachs JD. Macroeconomics and health: investing in health for economic development. Geneva: WHO; 2001.

21. Centers for Disease Control and Prevention (CDC). Economic impact of motor-vehicle crashes--United States, 1990. MMWR Morb Mortal Wkly Rep. 1993;42(23):443-8.

22. Gruenewald PJ, Remer L. Changes in outlet densities affect violence rates. Alcohol Clin Exp Res. 2006;30(7):1184-93.

23. Campbell CA, Hahn RA, Elder R, Brewer R, Chattopadhyay $\mathrm{S}$, Fielding $\mathrm{J}$, et al. The effectiveness of limiting alcohol outlet density as a means of reducing excessive alcohol consumption and alcohol-related harms. Am J Prev Med. 2009;37(6):556-69.

24. Rammohan V, Hahn RA, Elder R, Brewer R, Fielding J, Naimi TS, et al. Effects of dram shop liability and enhanced overservice law enforcement initiatives on excessive alcohol consumption and related harms two community guide systematic reviews. Am J Prev Med. 2011;41(3):334-43.

25. Task Force on Community Preventive Services. Recommendations on dram shop liability and overservice law enforcement initiatives to prevent excessive alcohol consumption and related harms. Am J Prev Med. 201;41(3):344-6.

26. Elder RW, Lawrence B, Ferguson A, Naimi TS, Brewer RD, Chattopadhyay SK, et al. The effectiveness of tax policy interventions for reducing excessive alcohol consumption and related harms. Am J Prev Med. 2010;38(2):217-29.
27. Task Force on Community Preventive Services. Increasing alcoholic beverage taxes is recommended to reduce excessive alcohol consumption and related harms. Am J Prev Med. 2010;38(2):230-2.

28. Elder R, Lawrence B, Janes G, Brewer R, Toomey T, Hingson R, et al. Enhanced enforcement of laws prohibiting sale of alcohol to minors: systematic review of effectiveness for reducing sales and underage drinking. Transportation Research Circular. 2007;E-C(123):181-8.

29. Dinh-Zarr T, Goss C, Heitman E, Roberts I, DiGuiseppi C. Interventions for preventing injuries in problem drinkers. Cochrane Database Syst Rev. 2004(3):CD001857.

30. Dinh-Zarr T, DiGuiseppi C, Heitman E, Roberts I. Interventions for preventing injuries in problem drinkers. Cochrane Database Syst Rev. 2000(2):CD001857.

31. Fiestas F, Ponce J, Gallo C, Bustamante I, Ordonez C, Mazzotti G. Factores predictores de uso problemático de alcohol en personas atendidas en una sala de emergencia. Rev Peru Med Exp Salud Publica. 2011;28(1):54-61.

32. Snyder LB, Milici FF, Slater M, Sun H, Strizhakova Y. Effects of alcohol advertising exposure on drinking among youth. Arch Pediatr Adolesc Med. 2006;160(1):18-24.

33. Saffer $\mathrm{H}$. Alcohol advertising bans and alcohol abuse: an international perspective. J Health Econ. 1991;10(1):65-79.

34. Anderson P, de Bruijn A, Angus K, Gordon R, Hastings G. Impact of alcohol advertising and media exposure on adolescent alcohol use: a systematic review of longitudinal studies. Alcohol Alcohol. 2009;44(3):229-43.

35. Ditter SM, Elder RW, Shults RA, Sleet DA, Compton R, Nichols JL. Effectiveness of designated driver programs for reducing alcohol-impaired driving: a systematic review. Am J Prev Med. 2005;28(5 Suppl):280-7.

\section{Correspondencia: Fabián Fiestas}

Dirección: Cápac Yupanqui 1400, Lima 11, Perú

Teléfono: (511) 985765743

Correo electrónico: ffiestas@epi.msu.edu

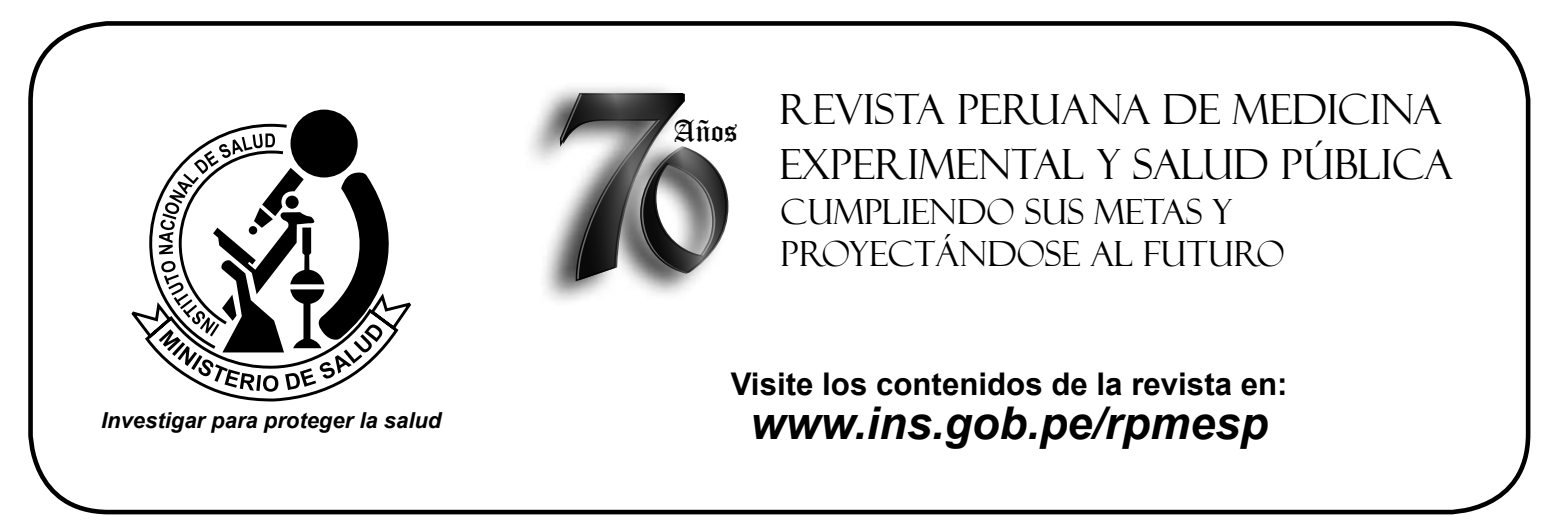

